\title{
Avaliação dermatológica de agentes comunitários de saúde sujeitos à fotoexposição em região tropical do Brasil
}

\author{
Dermatological evaluation of community health agents subjected to sun exposure \\ in a Brazilian tropical region
}

\section{Fernando Costa Araújo ${ }^{1} \bowtie$, Bruna Ranyelle de Marinho Sousa ${ }^{2}$, Glereston Gomes Leite ${ }^{3}$, Leidian Coelho de Freitas ${ }^{4}$, Edna Lamar da Costa Lemos ${ }^{5}$, Carla Andrea Avelar Pires ${ }^{6}$}

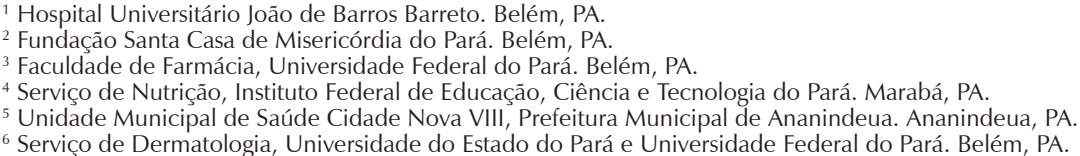

\section{RESUMO}

Objetivos: Investigar lesões de pele relacionadas ou agravadas com a exposição solar em agentes comunitários de saúde de dois municípios da região metropolitana de Belém, estado do Pará, relacionando-as com algumas variáveis sociodemográficas, e avaliar o uso de proteção à exposição solar

Métodos: O delineamento adotado foi um estudo transversal, incluindo agentes de saúde de 11 Unidades Saúde da Família de Belém e Ananindeua selecionados por amostra não probabilística de conveniência. Todos os indivíduos responderam a um questionário e foram submetidos a uma consulta dermatológica que avaliou presença de lesões de pele e variáveis relacionadas à fotoexposição e perfil sociodemográfico dos trabalhadores. As hipóteses foram avaliadas pelos testes qui-quadrado, exato de Fisher com correção de Yates e t de Student. Estabeleceu-se como significante $o$ valor de $\mathrm{p} \leq 0,05$.

Resultados: Foram avaliados 83 profissionais, dos quais a maioria pertencente ao gênero feminino (75,9\%), na faixa etária entre os 30 e 40 anos $(44,6 \%)$, solteiros $(47 \%)$, com o ensino médio completo $(69,8 \%)$ e renda familiar entre um a dois salários mínimos $(67,5 \%)$, atuando na profissão há mais de 10 anos (48,2\%). Dentre os entrevistados, $83 \%$ referiam exposição solar diária em sua atividade laborativa, $47 \%$ por mais de quatro horas, e $73,5 \%$ se expunham ao sol no horário mais crítico, entre as $10 \mathrm{~h}$ e $16 \mathrm{~h}$, sendo que $56,6 \%$ faziam uso de algum tipo de proteção contra a exposição solar e 27,7\% afirmaram usar protetor solar diariamente. Alguma alteração de pele relacionada à exposição solar foi encontrada em $81(97,6 \%)$ trabalhadores. Melanose solar foi o diagnóstico mais frequente.

Conclusões: Lesões de pele foram frequentes nesta amostra de agentes comunitários de saúde, e a exposição solar laboral parece ter tido um impacto significativo na sua ocorrência. Verificou-se que os participantes apresentavam dificuldade de adesão a fatores de fotoproteção. Políticas e ações de saúde ocupacional com ênfase na prevenção de agravos relacionados ao trabalho dos agentes comunitários de saúde devem ser estimuladas.

DESCRITORES: saúde do trabalhador; dermatologia; protetores solares; medicina de família e comunidade.

\section{ABSTRACT}

Aims: To investigate skin lesions associated with or aggravated by sun exposure in community health workers in two municipalities in the metropolitan region of Belém, State of Pará, Brazil, relating the lesions to some sociodemographic variables, and to assess the use of sun protection.

Methods: A cross-sectional study was carried out with 11 Family Health Centers in Belém and Ananindeua selected by non-probabilistic convenience sampling. All workers answered a questionnaire and were clinically examined for the presence of skin lesions caused by sun exposure, in addition to having sun exposure variables and their sociodemographic profile assessed. The data were analyzed by the chi-square test, Fisher's exact test with Yates' correction, and Student's t test. A p value $\leq 0.05$ was considered to be statistically significant.

Results: A total of 83 workers were assessed, most of whom were female (75.9\%), aged between 30 and 40 years (44.6\%), single (47\%), with completed high school education (69.8\%), and family income between 1 and 2 minimum wages $(67.5 \%)$, working in the profession for over 10 years $(48.2 \%)$. Among the respondents, $83 \%$ reported daily sun exposure in their working activity, $47 \%$ were exposed for more than four hours, and $73.5 \%$ were exposed to the sun at the most critical time (between 10 and 4 o'clock). 56.6\% used some kind of protection against sun exposure, and $27.7 \%$ reported using sunscreen on a daily basis. The dermatologic evaluation also revealed that $97.6 \%$ of workers had many skin lesions caused by sun exposure. Melanosis was the most common diagnosis.

Conclusions: Skin lesions were frequent among community health workers and sun exposure during working hours appears to have played a remarkable role. Workers showed difficulty adopting protective behaviors. Occupational health policies and actions targeted at the prevention of aggravating factors among community health workers should be encouraged.

KEYWORDS: occupational health; dermatology; sunscreening agents; family practice. 
Abreviaturas: ACS, agente comunitário de saúde; PACS, Programa de Agentes Comunitários de Saúde; UV, ultravioleta.

\section{INTRODUÇÃO}

O Agente Comunitário de Saúde (ACS) é um profissional que atua na atenção primária em saúde, especialmente no Programa de Agentes Comunitários de Saúde (PACS) e na Estratégia Saúde da Família. O ACS compõe a equipe de saúde, sendo responsável por uma microárea inclusa na área definida para sua equipe. Esse profissional busca desenvolver ações para a integração entre a equipe de saúde e a população. Geralmente reside na comunidade em que desempenha o seu trabalho, vivenciando os problemas e definindo um envolvimento pessoal diferenciado com questões que comprometem a saúde e o bem-estar das famílias acompanhadas por ele $[1,2]$.

Os ACS atuam nas equipes de Saúde da Família realizando atividades externas à unidade de saúde, expondo-se por longos períodos de tempo à radiação solar, estando sujeitos a inúmeros problemas ocasionados pela exposição ao sol [3]. Lesões dermatológicas podem expressar diversas condições clínicas que afetam a qualidade de vida no trabalho, além de representarem condições de morbimortalidade relacionadas à prática laboral. O entendimento dessa realidade, com vistas à difusão de medidas preventivas globais, representa importante passo para a promoção da saúde e proteção específica da doença no trabalhador.

Dentre as doenças de pele a que podem estar expostos os ACS, observa-se um grupo de patologias definidas como fotodermatoses: quadros cutâneos causados ou influenciados pela exposição à luz solar, com alterações inflamatórias (fotodermites) ou degenerativas. As fotodermatoses compreendem dois grupos distintos: o primeiro, fotodermatoses por irritação primária, são as reações cutâneas imediatas por superexposição à luz solar ou tardias por exposição crônica ou prolongada. O segundo grupo, fotodermatoses por sensibilização, reúne as reações cutâneas decorrentes da interação da luz solar com agentes fotossensibilizantes exógenos (contactantes) ou endógenos (endoctantes) e compreende as reações de fototoxicidade ou fotoalergia [4].

Dada a escassez de pesquisas direcionadas aos ACS no âmbito das doenças ocupacionais e tendo em vista que essa categoria profissional está sujeita a vários problemas acarretados pela exposição excessiva ao sol, o objetivo deste trabalho foi investigar lesões de pele relacionadas à exposição solar em ACS de dois municípios da região metropolitana de Belém, estado do Pará, e descrever brevemente o perfil sociodemográfico desses profissionais.

\section{MÉTODOS}

Foi realizado um estudo transversal, com indivíduos que atuam como ACS pelo programa Estratégia Saúde da Família nos municípios de Ananindeua e Belém, Pará, nos meses de agosto e setembro de 2013. A população amostral foi selecionada por amostragem não probabilística de conveniência, incluindo profissionais que tivessem disponibilidade de participação da pesquisa na unidade de saúde em datas pré-agendadas.

Foram incluídos na pesquisa ACS que atuassem na profissão há pelo menos seis meses e que estivessem desenvolvendo atividade laboral regular no período da coleta dos dados, bem como concordassem plenamente a participar do estudo e assinassem o Termo de Consentimento Livre e Esclarecido aprovado pelo Comitê de Ética em Pesquisa com Seres Humanos, o qual foi previamente lido e explicado aos sujeitos da pesquisa. Dessa forma, não foram incluídos os ACS que estivessem ausentes do trabalho em licença de qualquer natureza no período estabelecido da coleta de dados.

O estudo foi realizado em 11 Unidades de Saúde da Família da Região Metropolitana de Belém, das quais oito estão localizadas no município de Ananindeua (Cidade Nova IV, Cidade Nova VI, Cidade Nova VII, Cidade Nova VIII, Coqueiro I, Coqueiro III, Icuí e Stélio Maroja) e três estão localizadas na capital do estado, Belém (Canal do Pirajá, Paraíso dos Pássaros e Mangueirão). Os dados foram coletados por meio de um questionário semiestruturado, no próprio local de trabalho dos profissionais, que, posteriormente, foram convidados a participar de uma consulta médica dermatológica em ambulatórios da própria unidade de saúde em que trabalham, visitada por uma médica dermatologista em data pré-estabelecida com os entrevistados.

A avaliação dermatológica constou de um exame clínico detalhado e descrição das lesões observadas, acompanhada de uma anamnese dirigida aos achados dermatológicos, em que foram investigados sinais e sintomas subjetivos, história da lesão e antecedentes pessoais e familiares. Realizou-se, ainda, a comparação do aspecto da pele de uma parte do corpo mais exposta à radiação solar com a de outra mais protegida, o que foi utilizado para definir a presença de fotoenvelhecimento (superfície nodular áspera e 
espessa, repleta de manchas e de rugas acentuadas) que difere do envelhecimento cronológico da pele (textura mais lisa, ligeiramente atrofiada, com rugas discretas e sem manchas). Para estas análises, utilizou-se a escala de fototipos cutâneos proposta por Fitzpatrick [5], que é dividida em seis classificações que vai de I (pele branca, que sempre queima, nunca bronzeia e é muito sensível) a VI (pele negra, que nunca queima, totalmente pigmentada e insensível ao sol), estando as populações de fototipos inferiores submetidas a maior risco de lesões cutâneas relacionadas à exposição solar.

Foram levantadas as variáveis relacionadas ao perfil sociodemográfico do trabalhador, ao tempo e à frequência da exposição solar, aos fatores de fotoproteção utilizados e lesões de pele relacionadas à fotoexposição. Foi avaliada a associação entre os tipos de lesões dermatológicas diagnosticados e as variáveis gênero e tempo de trabalho na profissão.

$\mathrm{O}$ presente trabalho baseou-se nas diretrizes e normas regulamentadoras contidas na resolução 466/12 do Conselho Nacional de Saúde, que trata de pesquisas envolvendo seres humanos, e seguiu as recomendações do Conselho Nacional de Ética em Pesquisa, sendo aprovado pelo Comitê de Ética em Pesquisa do Instituto de Ciências da Saúde da Universidade Federal do Pará, sob o parecer $\mathrm{n}$ o 358.075 .

Os resultados obtidos foram armazenados em planilhas eletrônicas, utilizando o software Microsoft Office Excel 2010, e analisados usando o software Epi Info ${ }^{\mathrm{TM}}$, versão 3.5.2. As variáveis contínuas foram analisadas pelo estudo de medidas de tendência central, como média e mediana, e por medidas de variabilidade, como coeficiente de variância e desvio-padrão. As hipóteses foram avaliadas pelos testes qui-quadrado, teste exato de Fisher com correção de Yates e teste $t$ de Student. Estabeleceu-se como significância o valor de $\mathrm{p} \leq 0,05$.

\section{RESULTADOS}

A amostra incluiu 83 profissionais, cujos dados do perfil sociodemográfico são sumarizados na Tabela 1. Dentre os entrevistados, $83 \%$ referiam exposição solar diária em sua atividade laborativa, $47 \%$ por mais de quatro horas e $73,5 \%$ se expunham ao sol no horário mais crítico, entre as 10 e 16h. A maioria dos inquiridos apresentava fototipo cutâneo III (indivíduos morenos claros) segundo a classificação de Fitzpatrick [5], e menos da metade dos entrevistados usava regularmente protetor solar, sendo a falta de hábito e dificuldades financeiras para a aquisição do produto apontadas como as principais justificativas para o não uso da fotoproteção química (Tabela 2 ).
Tabela 1. Frequência de variáveis sociodemográficas e tempo de serviço de 83 Agentes Comunitários de Saúde de Belém e Ananindeua, PA, Brasil, 2013.

\begin{tabular}{|c|c|c|}
\hline Variáveis & $\mathbf{n}$ & $\%$ \\
\hline \multicolumn{3}{|l|}{ Gênero } \\
\hline Feminino & 63 & 75,9 \\
\hline Masculino & 20 & 24,1 \\
\hline \multicolumn{3}{|l|}{ Faixa Etária } \\
\hline $20 \mid-30$ anos & 12 & 14,5 \\
\hline $30 \mid-40$ anos & 37 & 44,6 \\
\hline $40 \mid-50 a n o s$ & 18 & 21,7 \\
\hline $50 \mid-60 a n o s$ & 12 & 14,5 \\
\hline$\geq 60$ anos & 4 & 4,8 \\
\hline \multicolumn{3}{|l|}{ Estado Civil } \\
\hline Solteiro & 39 & 47,0 \\
\hline Casado/união estável & 35 & 42,2 \\
\hline Separado & 9 & 10,8 \\
\hline \multicolumn{3}{|l|}{ Grau de escolaridade } \\
\hline Ensino fundamental & 6 & 7,2 \\
\hline Ensino médio & 58 & 69,8 \\
\hline Ensino superior & 19 & 22,9 \\
\hline \multicolumn{3}{|l|}{ Renda familiar } \\
\hline 1 |--| 2 salários mínimos & 56 & 67,5 \\
\hline 3 |--| 5 salários mínimos & 24 & 28,9 \\
\hline >5 salários mínimos & 3 & 3,6 \\
\hline \multicolumn{3}{|l|}{ Tempo de Profissão } \\
\hline$<1$ ano & 15 & 18,1 \\
\hline $1 \mid-3$ anos & 16 & 19,3 \\
\hline $3 \mid-6$ anos & 5 & 6,0 \\
\hline $6 \mid-10$ anos & 7 & 8,4 \\
\hline$\geq 10$ anos & 40 & 48,2 \\
\hline Total & 83 & 100,0 \\
\hline
\end{tabular}

A avaliação dermatológica revelou que 81 $(97,6 \%)$ dos trabalhadores apresentaram algum tipo de alteração de pele causado ou agravado pela exposição solar. Nesse grupo, observa-se na que os principais diagnósticos dermatológicos constatados foram: melanose solar $(71,6 \%)$, melasma $(70,4 \%)$ e leucodermia solar $(53,1 \%)$. Não foram encontradas lesões sugestivas de malignidade (Tabela 3 ).

Análises de associação revelaram que a leucodermia solar e o melasma foram significativamente mais frequentes no gênero feminino. As mulheres apresentaram risco relativo 3,8 e 4,4 maior de apresentar melasma e leucodermia solar, respectivamente, quando comparadas a homens. Demais diagnósticos relacionados à exposição solar não mostraram preferência por gênero (Tabela 4).

Quando se agruparam os indivíduos pelo tempo de trabalho como ACS, observou-se que a ceratose 
Tabela 2. Frequência de variáveis relacionadas à exposição solar e fotoproteção em 83 Agentes Comunitários de Saúde de Belém e Ananindeua, PA, Brasil, 2013.

\begin{tabular}{|c|c|c|}
\hline Variáveis & $\mathbf{n}$ & $\%$ \\
\hline \multicolumn{3}{|l|}{ Frequência da exposição } \\
\hline Pouca exposição & 5 & 6,1 \\
\hline Esporádica & 9 & 10,8 \\
\hline Diária & 69 & 83,1 \\
\hline \multicolumn{3}{|l|}{ Horas diárias de exposição solar } \\
\hline De uma a duas & 12 & 14,5 \\
\hline De duas a quatro & 32 & 38,6 \\
\hline De quatro a cinco & 20 & 24,1 \\
\hline Mais de cinco & 19 & 22,9 \\
\hline \multicolumn{3}{|l|}{ Horário em que mais se expõe ao sol } \\
\hline Entre $7 \mathrm{~h}$ e $10 \mathrm{~h}$ & 21 & 25,3 \\
\hline Entre $10 \mathrm{~h}$ e $16 \mathrm{~h}$ & 61 & 73,5 \\
\hline Após 16h & 1 & 1,2 \\
\hline \multicolumn{3}{|l|}{ Fatores de Proteção utilizados* } \\
\hline Bonés & 12 & 25,5 \\
\hline Sombrinhas/Guarda-chuva & 33 & 70,2 \\
\hline Protetor solar & 23 & 48,9 \\
\hline Nenhumt & 36 & 43,3 \\
\hline \multicolumn{3}{|c|}{ Fatores que dificultam uso do Protetor Solar* } \\
\hline Esquecimento/falta de tempo & 25 & 30,1 \\
\hline Não gosta/não vê utilidade & 2 & 2,4 \\
\hline Falta de hábito & 29 & 34,9 \\
\hline Falta de dinheiro para adquirir & 29 & 34,9 \\
\hline Todos os citados & 4 & 4,8 \\
\hline Nenhum & 8 & 9,6 \\
\hline \multicolumn{3}{|l|}{ Fototipos $¥$} \\
\hline II & 11 & 13,3 \\
\hline III & 37 & 44,6 \\
\hline IV & 24 & 28,9 \\
\hline V & 11 & 13,3 \\
\hline Total & 83 & 100,0 \\
\hline
\end{tabular}

*Mais de uma escolha era permitida nestes tópicos, utilizando-se número total.

† Porcentagem em relação ao número total de entrevistados.

‡ Segundo Classificação de Fitzpatrick [5].

Tabela 3. Lesões dermatológicas causadas ou agravadas pela exposição solar encontradas em 83 Agentes Comunitários de Saúde de Ananindeua e Belém, PA, Brasil, 2013.

\begin{tabular}{|lcc|}
\hline \multicolumn{1}{c}{ Lesões dermatológicas } & n & \%* \\
\hline Eritema solar & 3 & 3,7 \\
\hline Fotoenvelhecimento & 29 & 35,8 \\
\hline Leucodermia solar & 43 & 53,1 \\
\hline Xerodermia & 12 & 14,8 \\
\hline Melanose solar & 58 & 71,6 \\
\hline Ceratose seborreica & 24 & 29,6 \\
\hline Melasma & 57 & 70,4 \\
\hline Efélides & 10 & 12,3 \\
\hline Cutis romboidal & 3 & 3,7 \\
\hline Telangiectasia & 13 & 16,0 \\
\hline Nenhuma lesão & 2 & 2,4 \\
\hline
\end{tabular}

*A soma das porcentagens é superior a $100 \%$ porque muitos apresentavam mais de uma lesão. seborreica foi mais frequente entre os indivíduos que atuavam há mais de três anos na profissão, os quais apresentaram cerca de 3,9 vezes mais chance de possuir tal diagnóstico. Observou-se, ainda, que indivíduos que trabalhavam há mais de 10 anos como ACS possuíam cerca de 3,3 vezes mais chance de apresentar melasma (Tabela 5). Demais diagnósticos não mostraram relação com o tempo de serviço

Não foi encontrada associação entre as horas de exposição solar e as lesões dermatológicas apresentadas. Quando analisado o uso de protetores físicos (bonés, sombrinhas, etc.) à exposição solar e o uso de fotoproteção química, também não houve associação relevante com os diagnósticos apresentados. Demais análises cruzadas não se mostraram relevantes.

\section{DISCUSSÃO}

Os ACS têm se mostrado personagens essenciais no contexto da promoção da saúde no âmbito da atenção básica, sendo protagonistas no que tange à troca de experiências entre os saberes populares e o conhecimento médico-científico, fortalecendo a aproximação entre a comunidade e os serviços de saúde [6]. Entretanto, a existência de agravos relacionados ao trabalho desses servidores da saúde, tema pouco explorado, evidencia um grande paradoxo no contexto do Sistema Único de Saúde, relacionado à necessidade de proteger a saúde de quem cuida [7].

O perfil dos profissionais avaliados neste estudo está em consonância ao perfil dos ACS estudados em três coortes, realizadas em municípios do noroeste paulista, Vitória, no Espírito Santo, e Porto Alegre, no Rio Grande do Sul, em que a maioria dos inquiridos pertencia ao gênero feminino, possuía ensino médio completo e apresentava baixa renda per capita ou familiar, sendo esta última variável apontada como um dos fatores de maior insatisfação em relação à profissão [8-10].

Mais de $80 \%$ dos indivíduos do presente estudo afirmaram expor-se ao sol diariamente, muitos por mais de quatro horas e mais de $70 \%$ no horário mais crítico, entre $10 \mathrm{~h}$ e $16 \mathrm{~h}$. Do mesmo modo, uma pesquisa realizada no interior do estado de Minas Gerais revelou que a maioria dos ACS ficava exposta por mais de cinco horas diárias à radiação solar, sendo a totalidade em horário crítico [3]. Essa alta exposição representa um risco potencial de lesões de pele, das mais simples, que causam danos estéticos, às mais complexas e que representam malignidade. Sabe-se que a exposição excessiva ao sol é o principal fator de risco do câncer de pele. 
Tabela 4. Frequência absoluta e relativa e análise da associação entre gênero e diagnósticos dermatológicos nos 81 Agentes Comunitários de Saúde que apresentaram lesões cutâneas. Belém e Ananindeua, PA, Brasil, 2013.

\begin{tabular}{|c|c|c|c|c|}
\hline Tipo de lesão dermatológica & Presença de lesão & $\begin{array}{c}\text { Feminino } \\
\mathbf{n}(\%)\end{array}$ & $\begin{array}{c}\text { Masculino } \\
\text { n (\%) }\end{array}$ & $\mathbf{p}$ \\
\hline \multirow{2}{*}{ Eritema Solar } & Sim & $2(3,2)$ & $1(5,3)$ & \multirow{2}{*}{$0,556^{*}$} \\
\hline & Não & $60(96,8)$ & $18(94,7)$ & \\
\hline \multirow{2}{*}{ Fotoenvelhecimento } & Sim & $21(33,9)$ & $8(42,1)$ & \multirow{2}{*}{$0,702^{\dagger}$} \\
\hline & Não & $41(66,1)$ & $11(57,9)$ & \\
\hline \multirow{2}{*}{ Leucodermia solar } & Sim & $38(61,3)$ & $5(26,3)$ & \multirow{2}{*}{0,015} \\
\hline & Não & $24(38,7)$ & $14(73,7)$ & \\
\hline \multirow{2}{*}{ Xerodermia } & Sim & $8(12,9)$ & $4(21,1)$ & \multirow{2}{*}{$0,294 *$} \\
\hline & Não & $54(87,1)$ & $15(78,9)$ & \\
\hline \multirow{2}{*}{ Melanose solar } & Sim & $45(72,6)$ & $13(68,4)$ & \multirow{2}{*}{$0,951^{\dagger}$} \\
\hline & Não & $17(27,4)$ & $6(31,6)$ & \\
\hline \multirow{2}{*}{ Ceratose seborreica } & Sim & $20(32,3)$ & $4(21,1)$ & \multirow{2}{*}{$0,516^{\dagger}$} \\
\hline & Não & $42(67,7)$ & $15(78,9)$ & \\
\hline \multirow{2}{*}{ Melasma } & Sim & $48(77,4)$ & $9(47,4)$ & \multirow{2}{*}{$0,026^{\dagger}$} \\
\hline & Não & $14(22,6)$ & $10(52,6)$ & \\
\hline \multirow{2}{*}{ Efélides } & Sim & $10(16,1)$ & $0(0,0)$ & \multirow{2}{*}{$0,057^{*}$} \\
\hline & Não & $52(83,9)$ & $19(100,0)$ & \\
\hline \multirow{2}{*}{ Cutis romboidal } & Sim & $3(4,8)$ & $0(0,0)$ & \multirow{2}{*}{$0,443 *$} \\
\hline & Não & $59(95,2)$ & $19(100,0)$ & \\
\hline \multirow{2}{*}{ Telangiectasia } & Sim & $10(16,1)$ & $3(15,8)$ & \multirow{2}{*}{$0,636^{*}$} \\
\hline & Não & $52(83,9)$ & $16(84,2)$ & \\
\hline
\end{tabular}

* Teste exato de Fisher.

${ }^{\dagger}$ Teste do Qui-quadrado com correção de Yates.

Tabela 5. Frequência absoluta e relativa e associação* entre tempo de serviço e diagnósticos dermatológicos em Agentes Comunitários de Saúde de Belém e Ananindeua, PA, Brasil, 2013.

\begin{tabular}{|c|c|c|c|c|}
\hline Tipo de lesão dermatológica & $\begin{array}{l}\text { Presença } \\
\text { de lesão }\end{array}$ & \multicolumn{2}{|c|}{$\begin{array}{c}\text { Tempo de Serviço } \\
\text { n (\%) }\end{array}$} & $\mathbf{p}^{+}$ \\
\hline \multirow{3}{*}{ Ceratose seborreica } & & $\geq 3$ anos & $<3$ anos & \multirow{3}{*}{0,037} \\
\hline & Sim & $20(38,5)$ & $4(13,8)$ & \\
\hline & Não & $32(61,5)$ & $25(86,2)$ & \\
\hline \multirow{3}{*}{ Melasma } & & $\geq 10$ anos & $<10$ anos & \multirow{3}{*}{0,034} \\
\hline & Sim & $33(82,5)$ & $24(58,5)$ & \\
\hline & Não & $7(17,5)$ & $17(41,5)$ & \\
\hline
\end{tabular}

* Mostrados apenas os que tiveram associação estatisticamente significativa.

† Teste do Qui-quadrado com correção de Yates.

O efeito cumulativo da radiação solar ultravioleta (UV) é responsável pelo aumento mundial de câncer de pele não-melanoma, uma categoria que inclui o carcinoma de células escamosas e os seus precursores (as ceratoses actínicas), bem como carcinoma de células basais. Câncer de pele não-melanoma é o tipo mais comum de câncer em áreas do mundo com uma população de pele clara. A associação entre exposição ocupacional a raios UV com doenças malignas da pele têm sido bem demonstrada em estudos recentes, que evidenciaram associações epidemiológicas consistentes entre exposição solar e aumento do risco para câncer de pele [11-13]. Entretanto, é sabido que além da exposição solar, fatores genéticos e história familiar de câncer de pele, além de idade mais avançada, contribuem para tais lesões [12-13], o que poderia justificar a ausência de lesões malignas na casuística estudada.

A necessidade do uso de protetores solares, também denominados fotoprotetores, é indiscutível, visto que problemas relacionados à radiação solar podem ser minimizados com o seu uso adequado [14], que inclui o emprego diário de filtro solar, sua aplicação 
20 minutos antes da exposição e reaplicações a cada duas horas [15]. Neste estudo, menos da metade dos profissionais avaliados fazia uso regular do protetor solar. Falta de dinheiro para adquirir o produto foi um motivo apontado por uma grande parte dos ACS para a dificuldade da adesão ao mesmo. Tendo em vista que a maior parte dos entrevistados possuía renda familiar média entre um e dois salários-mínimos e levando-se em conta o alto custo dos protetores solares, justifica-se, nesse estudo, a baixa adesão ao uso desse mecanismo de fotoproteção.

Em pesquisa realizada com carteiros da cidade de Botucatu, evidenciou-se que $87,88 \%$ dos entrevistados faziam uso de alguma medida de proteção à exposição solar, sendo que $72,41 \%$ utilizavam calça comprida, $68,96 \%$ faziam uso de boné e $52,38 \%$ utilizavam protetor solar diariamente [16]. Acreditase que a implantação de um programa de prescrição e distribuição de filtro solar, assim como dos demais equipamentos de proteção ao sol, possa contribuir para aumentar a aderência à fotoproteção pelos ACS.

Alguns inquéritos populacionais têm demonstrado que métodos de barreira física à exposição solar - tais como guarda-chuvas, óculos de sol, bonés/chapéus e roupas protetoras - são, depois do uso do filtro solar, as medidas diárias mais utilizadas para proteção solar [17,18]. Embora culturalmente bem aceitos e indicados como adjuvantes na fotoproteção, a eficácia de tais métodos isoladamente ainda carece de confirmação científica. Uma metanálise recente que se propôs a investigar o efeito de diversas estratégias de proteção solar na prevenção do câncer de pele nãomelanoma não identificou estudos que avaliassem a eficácia de medidas físicas de fotoproteção, como roupas, chapéus ou óculos de sol, na prevenção desses tumores [19].

Os principais diagnósticos dermatológicos causados ou agravados pela exposição solar descritos neste estudo foram: melanose solar, melasma e leucodermia solar. A melanose solar caracteriza-se por manchas hipercrômicas pequenas, de limites bem nítidos e número variado, que surgem no adulto geralmente após a quarta década, em áreas fotoexpostas, o que corrobora com este estudo, uma vez que a maior parte dos participantes encontrava-se com mais de 30 anos. A melanose solar também foi indicada como a dermatose mais frequente segundo a queixa secundária em uma casuística com pacientes da quarta idade (acima de 80 anos) [20]; foi a dermatose significativamente mais prevalente em indivíduos com mais de 60 anos de idade residentes em uma instituição filantrópica de longa permanência no município de Santos, seguida da ceratose seborreica [21]; e uma das três alterações mais frequentes na pele de pescadores profissionais de Recife, submetidos a radiação solar crônica prolongada [22].

O melasma é outra dermatose frequente no consultório dermatológico, caracterizada clinicamente por hipercromia adquirida simétrica, que ocorre exclusivamente em áreas fotoexpostas, geralmente em face. Mulheres em período fértil e de fototipos intermediários constituem as populações mais acometidas pelo melasma, o que justifica seu achado e sua prevalência neste estudo, dado que as mulheres representaram a maioria da casuística. Sua fisiopatogenia permanece desconhecida, porém há relação com fotoexposição, fatores hormonais, genéticos, doenças endócrinas e uso de medicamentos e cosméticos $[23,24]$.

Neste estudo identificou-se que os ACS eram em sua maioria mulheres, na quarta década de vida, que atuavam na profissão há mais de 10 anos. Lesões de pele foram muito frequentes nesta amostra, e a exposição solar laboral pode ter tido um impacto significativo na sua ocorrência. Outra possível explicação para o surgimento dessas lesões é a idade mais avançada dessas pacientes. Um estudo com trabalhadores latinos migrantes observou que a prevalência do melasma foi maior naqueles com idade superior a 31 anos [25].

Este estudo apresentou como principais limitações o tamanho amostral e a dificuldade de se estabelecer relações causais mais consistentes entre as lesões de pele e demais variáveis estudadas. Ainda assim, preocupa o fato de que os ACS, sob risco de doenças cutâneas pelo grau, tempo e período de exposição solar, apresentem tanta dificuldade de acesso e/ou de adesão a fatores de fotoproteção. Políticas e ações de saúde ocupacional com ênfase na prevenção de agravos relacionados ao trabalho como ACS devem ser estimuladas e fortalecidas. 
NOTA

Declaração de conflitos de interesse

Os autores declaram não haver conflitos de interesse relevantes ao conteúdo deste estudo.

\section{REFERÊNCIAS}

1. Baptistini RA, Figueiredo TAM. Agente comunitário de saúde: desafios do trabalho na zona rural. Ambient Soc. 2014;17(2):53-70. http://dx.doi.org/10.1590/S1414-753X2014000200005

2. Bezerra AFB, Espírito Santo ACG, Batista Filho M. Concepções e práticas do agente comunitário na atenção à saúde do idoso. Rev Saúde Pública. 2005;39(5):809-15. http://dx.doi.org/10.1590/S0034-89102005000500017

3. Lima AG, Silva AMM, Soares CEC, Souza RAX, Souza MCMR. Fotoexposição solar e fotoproteção de agentes de saúde em município de Minas Gerais. Rev Eletr Enf. 2010;12(3):478-82. http://dx.doi.org/10.5216/ree.v12i3.6156

4. Sampaio SAP, Rivitti EA. Dermatologia. $3^{\mathrm{a}}$ ed. São Paulo: Artes Médicas; 2008.

5. Fitzpatrick TB. The validity and practicality of sun-reactive skin types I through VI. Arch Dermatol. 1988;124(6):869-71. http://dx.doi. org/10.1001/archderm.1988.01670060015008

6. Nunes MO, Trad LB, Almeida BA, Homem CR, Melo MCIC. O agente comunitário de saúde: construção da identidade desse personagem híbrido e polifônico. Cad Saúde Pública. 2002;18(6):1639-46. http://dx.doi.org/10.1590/S0102-311X2002000600018

7. Vilela RAG, Silva RC, Jackson Filho JM. Poder de agir e sofrimento: estudo de caso sobre Agentes Comunitários de Saúde. Rev Bras Saúde Ocup. 2010;35(122):289-302. http://dx.doi.org/10.1590/S0303-76572010000200011

8. Santos KT, Saliba NA, Moimaz SAS, Arcieri RM, Carvalho ML. Agente comunitário de saúde: perfil adequado a realidade do Programa Saúde da Família?. Ciênc Saúde Coletiva. 2011;16 Suppl 1:1023-8. http://dx.doi.org/10.1590/S1413-81232011000700035

9. Barcellos CSN, Pandolfi M, Miotto MHMB. Perfil do Agente Comunitário de Saúde (ACS) de Vitória-ES. UFES Rev Odontol. 2006: $8(1): 21-8$.

10. Ferraz L, Aerts DRGC. O cotidiano de trabalho do agente comunitário de saúde no PSF em Porto Alegre. Ciênc Saúde Coletiva. 2005;10(2):347-55. http://dx.doi.org/10.1590/S1413-81232005000200012

11. Fartasch M, Diepgen TL, Schmitt J, Drexler H. The relationship between occupational sun exposure and non-melanoma skin cancer: clinical basics, epidemiology, occupational disease evaluation, and prevention. Dtsch Arztebl Int. 2012 Oct;109(43):715-20.

12. Schmitt J, Seidler A, Diepgen TL, Bauer A. Occupational ultraviolet light exposure increases the risk for the development of cutaneous squamous cell carcinoma: a systematic review and meta-analysis. Br J Dermatol. 2011;164(2):291-307. http://dx.doi.org/10.1111/j.13652133.2010.10118.x

13. Diepgen TL, Fartasch M, Drexler H, Schmitt J. Occupational skin cancer induced by ultraviolet radiation and its prevention. Br J Dermatol. 2012;167 Suppl 2:76-84. http://dx.doi.org/10.1111/j.1365-2133.2012.11090.x

14. Flor J, Davolos MR, Correa MA. Protetores solares. Quím Nova. 2007;30(1):153-8. http://dx.doi.org/10.1590/S0100-40422007000100027

15. Scarlett WL. Ultraviolet radiation: sun exposure, tanning beds, and vitamin D levels. What you need to know and how to decrease the risk of skin cancer. J Am Osteopath Assoc. 2003;103(8):371-5.

16. Popim RC, Corrente JE, Marino JAG, Souza CA. Câncer de pele: uso de medidas preventivas e perfil demográfico de um grupo de risco na cidade de Botucatu. Ciênc Saúde Coletiva. 2008;13(4):1331-6. http://dx.doi.org/10.1590/S1413-81232008000400030

17. Cheng S, Lian S, Hao Y, Kang N, Li S, Nie Y, Zhang F. Sun-exposure knowledge and protection behavior in a North Chinese population: a questionnaire-based study. Photodermatol Photoimmunol Photomed. 2010 Aug;26(4):177-81. http://dx.doi.org/10.1111/j.16000781.2010.00513.x

18. Bardini G, Lourenço D, Fissmer MC. Avaliação do conhecimento e hábitos de pacientes dermatológicos em relação ao câncer de pele. Arq Catarin Med. 2012;41:56-63.

19. Sánchez G, Nova J, Rodriguez-Hernandez AE, Medina RD, Solorzano-Restrepo C, Gonzalez J, Olmos M, Godfrey K, Arevalo-Rodriguez I. Sun protection for preventing basal cell and squamous cell skin cancers. Cochrane Database Syst Rev. 2016 Jul 25;7:CD011161. http:// dx.doi.org/10.1002/14651858.cd011161.pub2

20. Sittart JAS, Zanardi FHT. Prevalência das dermatoses em pacientes da 4a idade. Rev Soc Bras Clin Med. 2008;6(4):125-9.

21. Dinato SLM, Oliva R, Dinato MM, Macedo-Soares A, Bernardo WM. Prevalência de dermatoses em idosos residentes em instituição de longa permanência. Rev Ass Med Bras. 2008;54(6):543-7. http://dx.doi.org/10.1590/S0104-42302008000600020

22. Bezerra SMFMC, Sotto MN, Orii NM, Alves C, Duarte AJS. Efeitos da radiação solar crônica prolongada sobre o sistema imunológico de pescadores profissionais em Recife (PE), Brasil. An Bras Dermatol. 2011;86(2):222-33. http://dx.doi.org/10.1590/S036505962011000200004

23. Miot LDB, Miot HA, Silva MG, Marques MEA. Fisiopatologia do melasma. An Bras Dermatol. 2009;84(6):623-35. http://dx.doi. org/10.1590/S0365-05962009000600008

24. Ritter CG. Melasma extra-facial: avaliação clínica, histopatológica e imuno-histoquímica em estudo caso-controle [dissertation]. [Porto Alegre]: Faculdade de Medicina, Universidade Federal do Rio Grande do Sul; 2011. 83 p.

25. Pichardo R, Vallejos Q, Feldman SR, Schulz MR, Verma A, Quandt SA, Arcury TA. The prevalence of melasma and its association with quality of life in adult male Latino migrant workers. Int J Dermatol. 2009;48(1):22-6. http://dx.doi.org/10.1111/j.1365-4632.2009. 03778.x 